This is an electronic reprint of the original article. This reprint may differ from the original in pagination and typographic detail.

\author{
Author(s): Lahtinen, Elmeri; Kivijärvi, Lauri; Tatikonda, Rajendhraprasad; Väisänen, Ari; Rissanen, \\ Kari; Haukka, Matti
}

Title: $\quad$ Selective recovery of gold from electronic waste using 3D-printed scavenger

Year: $\quad 2017$

Version:

Please cite the original version:

Lahtinen, E., Kivijärvi, L., Tatikonda, R., Väisänen, A., Rissanen, K., \& Haukka, M. (2017). Selective recovery of gold from electronic waste using 3D-printed scavenger. ACS Omega, 2(10), 7299-7304. https://doi.org/10.1021/acsomega.7b01215

All material supplied via JYX is protected by copyright and other intellectual property rights, and duplication or sale of all or part of any of the repository collections is not permitted, except that material may be duplicated by you for your research use or educational purposes in electronic or print form. You must obtain permission for any other use. Electronic or print copies may not be offered, whether for sale or otherwise to anyone who is not an authorised user. 


\title{
Selective Recovery of Gold from Electronic Waste Using 3D-Printed Scavenger
}

\author{
Elmeri Lahtinen, ${ }^{\dagger}$ Lauri Kivijärvi, ${ }^{\dagger}$ Rajendhraprasad Tatikonda, ${ }^{\dagger}$ Ari Väisänen, ${ }^{*}{ }^{\dagger}$ Kari Rissanen, ${ }^{*},{ }^{\dagger},+0$ \\ and Matti Haukka*,†
}

${ }^{\dagger}$ Department of Chemistry and ${ }^{\ddagger}$ Nanoscience Center, University of Jyväskylä, P.O. Box 35, FI-40014 Jyväskylä, Finland

\section{Supporting Information}

ABSTRACT: Around 10\% of the worldwide annual production of gold is used for manufacturing of electronic devices. According to the European Commission, waste electric and electronic equipment is the fastest growing waste stream in the European Union. This has generated the need for an effective method to recover gold from electronic waste. Here, we report a simple, effective, and highly selective nylon-12-based three-dimensional (3D)-printed scavenger objects for gold recovery directly from an aqua regia extract of a printed circuit board waste. Using the easy to handle and reusable 3Dprinted meshes or columns, gold can be selectively captured both in a batch and continuous flow processes by dipping the scavenger into the solution or passing the goldcontaining solution through the column. The possibility to optimize the shape, size, and flow properties of scavenger objects with $3 \mathrm{D}$ printing enables the gold scavengers to match the requirements of any processing plants.

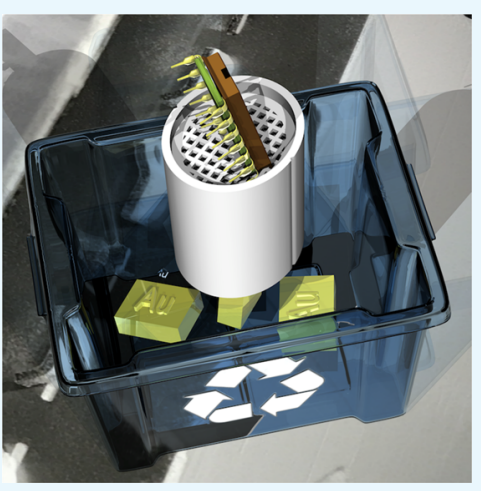

\section{INTRODUCTION}

The development of three-dimensional (3D) printing techniques and printing materials has been booming over the past decades. ${ }^{1}$ Materials from resins to metals can nowadays be printed to produce complex objects with precise dimensions and desired mechanical properties. The focus of interest is slowly expanding toward 3D-printed objects that possess additional physical or chemical functionalities. Currently, the 3D printing technique has been utilized for antimicrobial composites $^{2}$ and ion-exchange membranes, ${ }^{3,4}$ as well as for $\mathrm{pH}$ sensitive and catalytically active materials. ${ }^{5-7}$ Even polymerbonded rare-earth metal magnets ${ }^{8}$ and quantum dot lightemitting diodes have been successfully printed. ${ }^{9}$ Despite the progress in the field of functional 3D objects, the true potential of the technique has not been fully exploited, e.g., in chemistry. The use of 3D printing techniques in production of continuous flow reactors, chromatographic, or ion-exchange columns using an active material would open up a completely new level of control, e.g., of the reagent flow. If the conventional columns filled with active powder or beads are replaced with a column in which the adsorbent is also printed, practical pitfalls, such as unfavorable adsorbent packing and uneven eluent flow through the column, could be completely avoided. In the current work, we have used this approach in hydrometallurgy of electronic waste to prepare an efficient, selective, and simple collector for capturing gold from a complex mixture of dissolved metal ions.

Gold is widely used in the electronic industry, and with the waste electric and electronic equipment (WEEE) stream generated in the European Union predicted to be over 12 million tons by $2020,{ }^{10}$ an effective recovery method for gold is needed. Currently, used gold recovery methods include physical, pyrometallurgical, or hydrometallurgical treatments, or their combinations. ${ }^{11-14}$ However, most of these methods suffer from environmental risks and from the need for extensive preprocessing of the material. ${ }^{15-17}$ Hydrometallurgical processes have proven to be successful for leaching ${ }^{13,18,19}$ of gold from WEEE, but the recovery of gold from solution is often laborious, containing several steps. ${ }^{12,20,21}$ After the leaching, ion-exchange resins are often being used as an adsorbent for the gold, as has been widely reported. ${ }^{18,22-26}$ There have also been reports of polymers, such as polypyrrole and polyaniline, being used to recover gold. ${ }^{27-29}$ Because of lack of selectivity of the adsorbents and various other recovery methods, extensive preprocessing of the sample is often required. ${ }^{17,30}$ Typical scavenger materials used to adsorb the dissolved gold consist of small particles and hence a filtration system is often required, either to recover the used adsorbent or to purify the solution stream of remaining particles. The above difficulties make recycling the adsorbent challenging or even impossible.

These problems can be avoided by using $3 \mathrm{D}$ printing. ${ }^{31}$ The scavenger material is printed in a form of a column or a mesh, and the ion-containing solution is either flowed through it or the object is simply dipped into the solution. The captured metal ions can then be recovered by elution with a suitable solution, after which the scavenger is reusable for metal capturing. We used inexpensive $50 \mu \mathrm{m}$ nylon-12 (N12) powder for printing scavengers and tested these scavengers to selectively adsorb gold from aqua regia solutions containing up to 500 times higher concentrations of other metal ions. The binding of gold as $\left[\mathrm{AuCl}_{4}\right]^{-}$on nylon has been previously

Received: August 20, 2017

Accepted: October 17, 2017

Published: October 27, 2017 


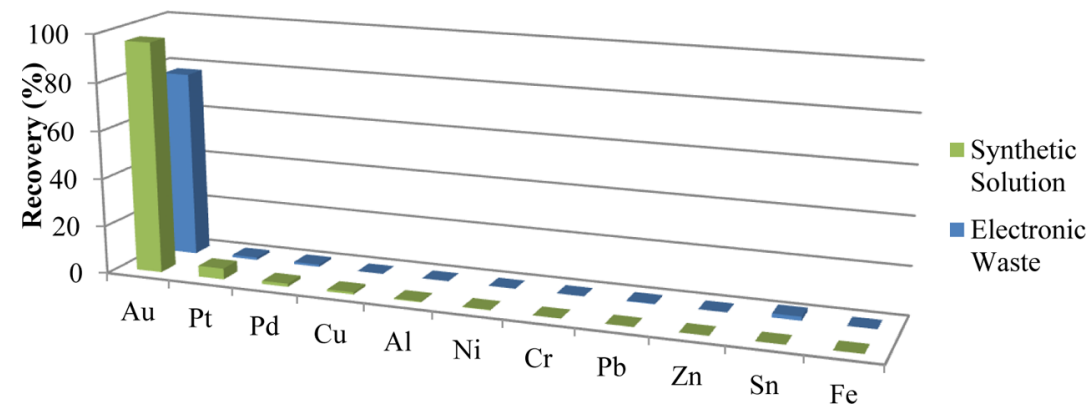

Figure 1. Recovery percentages of the metals by nylon- 12 powder from the synthetic $5 \% \mathrm{HCl}$ test solution (green) and from a 1:1 diluted aqua regia leached true electronic waste sample (blue). As can be seen, nylon-12 retains its efficiency and selectivity even in harsh oxidizing conditions.
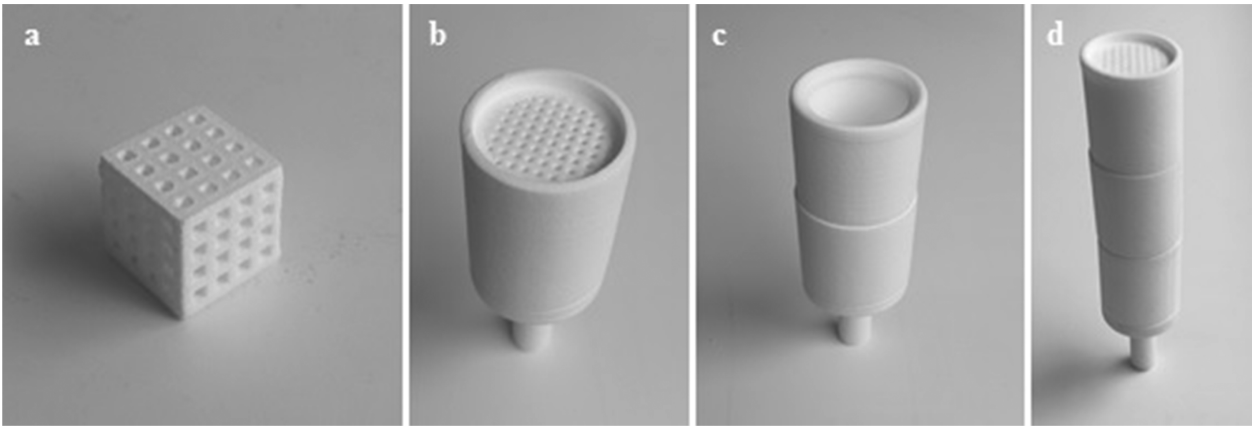

Figure 2. (a) Three-dimensionally printed cube-shaped mesh with dimensions of $18 \mathrm{~mm}$ x $18 \mathrm{~mm}$ x $18 \mathrm{~mm}$ used for batch tests. (b) Structure of the scavenger unit (length $35 \mathrm{~mm}$ and diameter $27 \mathrm{~mm}$ ) with a flow restricting funnel used for continuous flow processes (c). The complete column consists of three interlocking parts: the funnel at the bottom, the main gold scavenging unit in the middle and the reservoir cylinder at the top used for loading the sample (c). (d) An extended column with three scavenging units and the funnel.

noted, ${ }^{32}$ but to our knowledge, the excellent selectivity of nylon toward gold has not been reported. The binding mechanism between the amide group and $\left[\mathrm{AuCl}_{4}\right]^{-}$has been predicted to be related to hydrogen bonding between the hydrogen of the protonated amide group and the chlorides of the gold complex. ${ }^{33}$ The cause for selectivity toward gold could potentially be because of -1 charge of the complex and square planar geometry of the $\left[\mathrm{AuCl}_{4}\right]^{-}$. The only other species possessing square planar geometries in the solution would be chloride complexes of $\mathrm{Pt}(\mathrm{II})$ and $\mathrm{Pd}(\mathrm{II})$. These, however, would have a charge of -2 . The selectivity of the material will be investigated further in future by using molecular modeling.

\section{RESULTS AND DISCUSSION}

To find out the impact of $3 \mathrm{D}$ printing by the selective laser sintering (SLS) technique on the properties of nylon-12, we first tested the gold-capturing efficiency of the powder. The selectivity was tested using a solution mimicking the high concentrations of other metals typically present in acid-leached electronic waste (100 ppm of $\mathrm{Ni}, \mathrm{Zn}, \mathrm{Fe}$, and $\mathrm{Cu}$ and $50 \mathrm{ppm}$ of $\mathrm{Al}, \mathrm{Cr}, \mathrm{Pb}$, and $\mathrm{Sn}$ along with $5 \mathrm{ppm}$ of $\mathrm{Pd}, \mathrm{Pt}$, and $\mathrm{Au}$ in $5 \%$ hydrochloric acid). The $10 \mathrm{~mL}$ samples were treated with 50 $\mathrm{mg}$ of N12 powder, and the slurry was stirred at room temperature for $4 \mathrm{~h}$ after which the powder was filtered off. The PerkinElmer inductively coupled plasma optical emission spectrometry (ICP-OES) Optima $8300 \mathrm{DV}$ was used to determine the metal-ion concentrations in solution before and after the adsorption experiments. All in all, $96.4 \%$ of gold was adsorbed by the N12 powder together with $4.6 \%$ of the platinum, whereas noticeable adsorption of any of the other metals was not observed (Figure 1, green columns). To compare adsorption properties of different nylon polymers, nylon-11 (N11) and one of the most commonly used commercial grade of nylon, nylon-6,6 (N66), were also tested under the same conditions as that of N12. The N11 behaves much like N12, showing comparable selectivity with N12, but N66 was found to be less selective, adsorbing $25.6 \%$ of platinum and $13.8 \%$ of palladium along with $93.6 \%$ of the gold (Table S1). As nylon polymers are extensively used by the clothing industry, the typical fabric used, for example, for sport socks ( $82 \%$ of nylon, $16 \%$ of polypropylene, and $2 \%$ of elastane), was also tested and found to have similar properties as those of the N66 powder (Table S1). The comparisons indicate clearly that the highest selectivity can be achieved by using longer carbon chain nylon polymers N12 and N11.

To test the N12 powder with real electronic waste, a sample of aqua regia leached printed circuit boards (PCBs) was used. The PCB sample used contained $25000 \mathrm{mg} \mathrm{L}^{-1}$ copper, 2700 $\mathrm{mg} \mathrm{L}^{-1}$ iron, $1700 \mathrm{mg} \mathrm{L}^{-1}$ zinc, $1600 \mathrm{mg} \mathrm{L}^{-1}$ nickel, and 850 $\mathrm{mg} \mathrm{L}^{-1}$ tin, along with high concentrations of other metal ions. The gold concentration in PCB was $73 \mathrm{mg} \mathrm{L}^{-1}$. PCB leachate also contains silver, but the presence of chloride in the leaching solution accompanied by dilution leads to precipitation of silver chloride. Therefore, changes in silver concentration were not monitored. Silver precipitate can be simply filtered off before using a nylon scavenger. In N12 powder adsorption experiments, the PCB samples were diluted in a $1: 1$ ratio with ultrapure water and then stirred with $50 \mathrm{mg}$ of N12 for $4 \mathrm{~h}$. From the PCB sample, $77.8 \%$ of the gold was adsorbed, whereas tin $(1.6 \%)$ was the only other metal-ion adsorbed (Figure 1, blue columns), showing the excellent selectivity of N12 toward gold. The maximum gold-adsorbing capacity of the $\mathrm{N} 12$ powder was tested by mixing $25 \mathrm{mg}$ of $\mathrm{N} 12$ with $10 \mathrm{~mL}$ of a solution containing $200 \mathrm{ppm}$ of $\left[\mathrm{AuCl}_{4}\right]^{-}$in $5 \%$ hydrochloric 

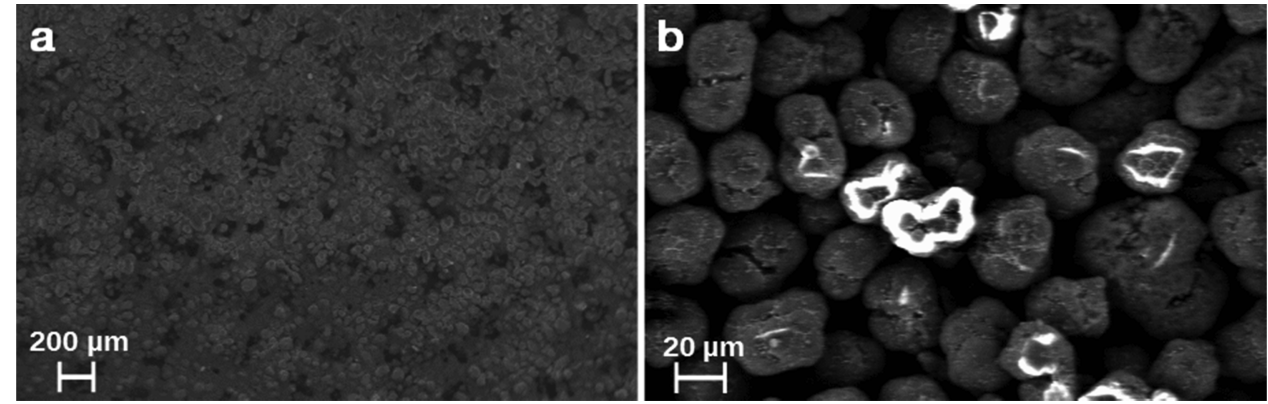

Figure 3. Scanning electron microscopy (SEM) images of the surface of solid objects printed with low laser power.

acid for $24 \mathrm{~h}$. A capacity higher than $66 \mathrm{mg}$ of gold per $1 \mathrm{~g}$ of N12 powder was observed.

The actual 3D-printed scavengers were prepared by using N12 powder and a selective laser sintering (SLS) 3D printing technique. Both cube-shaped meshes for batch processes and column-shaped scavengers for continuous flow processes were printed (Figure 2). The selectivity and recovery capability of the 3D-printed mesh (Figure 2a) in a batch process were tested using the same test solution as that for the N12 powder. Soaking the mesh into the test solution for $4 \mathrm{~h}$ yielded gold recovery of $90.4 \%$ with no noticeable amounts of other metals adsorbed into the mesh.

The continuous flow behavior of the short 3D-printed column shown in Figure $2 \mathrm{~b}$ (35 mm long scavenger part with $27 \mathrm{~mm}$ diameter) was tested by passing the test solution through the column. With the tested short column, the flow rate was ca. $3 \mathrm{~mL} \mathrm{~s}^{-1}$. The high flow rate leads to overall contact times of ca. 3-5 s with a $10 \mathrm{~mL}$ sample. Even with this very short contact time, the tested scavenger adsorbed $27.2 \%$ of gold with only traces of other metals. With a column consisting of three scavenger units (total length $105 \mathrm{~mm}$ ) 47.8\% and with a column of 10 scavenger units (total length $350 \mathrm{~mm}$ ), $82.7 \%$ of gold was captured. Even in the last case, the contact time remained very short (30s). The results can be even further improved by adjusting the flow rate through to the column. This can be done either by restricting the flow rate by using a valve or by redesigning and optimizing the flow channels in the scavenger units. The total recovery percentage can also be increased by recycling the solution through the column.

The capacity of the 3D-printed objects depends on the structure of the object and the printing parameters used. Because the specific surface area and capacity is dependent on the shape, size, and the design of the flow channels, it is difficult to give a simple figure for the capacity of the printed scavenger. Therefore, we tested the capacity of small solid rectangular printed plates with no elaborated pore structure to get a limiting value for the capacity. The capacity of these solid objects ranged from 13 to $18 \mathrm{mg}$ per $1 \mathrm{~g}$ of the $3 \mathrm{D}$-printed object. However, the surface area and thereof capacity of the 3D-printed object can be improved considerably by careful design of the surface structure.

One of the advantages of the scavenger objects is that gold can be nearly quantitatively removed from them by a simple elution process. Elution can be carried out by using nitric acid as eluent if acidic conditions are preferred (see the Methods section). Effective elution can also be obtained by using organic eluents such as diethylene glycol dibutyl ether. In both cases, the 3D-printed scavenger is fully reusable after gold has been removed.
The results show that $3 \mathrm{D}$ printing retains the excellent gold recovering properties of nylon-12. Obviously, 3D printing reduces the surface area of the object compared to the same amount of powder. However, the surface structure of the printed objects can be controlled up to a point by adjusting the 3D printing conditions: low laser power will produce an object with higher porosity and larger surface area (Figure 3). High laser power will increase the durability of the object but reduce the surface area, as the sintering of the material is more extensive (Figure S1).

The main advantage of the use of $3 \mathrm{D}$-printed scavengers is that the gold can be separated directly from the diluted leachate with no tedious preprocessing steps. Scavengers can be easily scaled (Figure S2c) and modified accordingly to meet the users' requirements even up to the industrial scale. It is expected that by using other printable polymers with other functional groups or hybrid materials, other metals can also be captured by using $3 \mathrm{D}$-printed scavengers. In such a case, scavenger modules with different selectivity could be linked together to obtain multimetal scavengers with detachable ion-specific modules. Because the 3D-printed scavengers are highly selective, they can be used for recovering gold from leachates originating from sources other than electronic waste as well. In general, the use of chemically functional printing materials can extend considerably the use of $3 \mathrm{D}$ printing in manufacturing of chemically active devices.

\section{METHODS}

Chemicals and Solvents. Synthetic solutions were prepared from stock spectroscopy standard solutions (1000 ppm) purchased from PerkinElmer. Nylon-12 was purchased from EOS and Advanc3d Materials and was in the form of powder, with an average particle diameter of $50 \mu \mathrm{m}$. Nylon-11 powder having an average particle diameter of $50 \mu \mathrm{m}$ was purchased from Advanc3d Materials. Nylon-6,6 was purchased from Sigma-Aldrich and had an average particle diameter of $15-20 \mu \mathrm{m}$. Nitric acid ( $\geq 65 \%)$ and hydrochloric acid $(\geq 37 \%)$ were purchased from Sigma-Aldrich. All of the chemicals were used as received. High-purity water of $18.2 \mathrm{M} \Omega \mathrm{cm}$ resistivity produced by a Purelab Ultra water purification system supplied by Elga (Buckinghamshire, U.K.) was used in all experiments. No unexpected or unusually high safety hazards were encountered.

Sample Preparation. The sample solution for the synthetic tests was prepared by diluting the $1000 \mathrm{ppm}$ PerkinElmer spectroscopy standards. The solution prepared contained $100 \mathrm{ppm}$ of $\mathrm{Ni}, \mathrm{Zn}, \mathrm{Fe}$, and $\mathrm{Cu}$ and $50 \mathrm{ppm}$ of $\mathrm{Al}, \mathrm{Cr}$, $\mathrm{Pb}$, and $\mathrm{Sn}$ along with $5 \mathrm{ppm}$ of $\mathrm{Pd}, \mathrm{Pt}$, and $\mathrm{Au}$ in $5 \%$ hydrochloric acid. The electronic waste sample was prepared by 
milling printed circuit boards (PCBs) down to fine particles and ashing them for $4 \mathrm{~h}$ at the temperature of $950{ }^{\circ} \mathrm{C}$. One gram of the ashed sample was placed in a $50 \mathrm{~mL}$ centrifuge tube along with $10 \mathrm{~mL}$ of aqua regia. Ultrasound-assisted leaching was performed at room temperature using six cycles lasting 3 min each. Between the cycles, the pressure was released from the reaction containers. The ultrasound water bath used was Elma Elmasonic P. Leached samples were filtered (Whatman 41) and diluted in a 1:1 ratio with ultrapure water.

Recovery from Synthetic Solution. A $10 \mathrm{~mL}$ sample of the synthetic solution was placed in a $15 \mathrm{~mL}$ centrifuge tube. Fifty milligrams of nylon-12 powder was added into the tube. The tube was sealed and then stirred using Stuart SF1 at 500 osc $\mathrm{min}^{-1}$ for $4 \mathrm{~h}$ at room temperature. Nylon-12 was removed from the sample solution by filtration (Whatman 41). The same procedure was used for testing nylon-11 (N11), nylon-6,6 (N66), and the fabric samples. All of the tests discussed in this article were performed in triplicate. Recovery percentages of the metals with significant decrease in concentration are presented in Table S1.

Recovery from Synthetic Solution Using Acid-Washed N12 Powder. A $1 \mathrm{~g}$ sample of the N12 powder was placed in a glass column and $200 \mathrm{~mL}$ of $10 \%$ aqua regia, followed by 200 $\mathrm{mL}$ of water being passed through it. The N12 powder was dried at $105{ }^{\circ} \mathrm{C}$ for $4 \mathrm{~h}$ and was then used for adsorption tests. Ten milliliters of the synthetic solution was placed in a $15 \mathrm{~mL}$ centrifuge tube. Washed and dried N12 powder $(50 \mathrm{mg}$ ) was added into the tube. The tube was sealed and then agitated using Stuart SF1 at 500 osc $\mathrm{min}^{-1}$ for $4 \mathrm{~h}$ at room temperature. Nylon-12 was removed from the sample solution by filtration (Whatman 41). Recovery percentages of the metals with significant decrease in concentrations are presented in Table S1.

Recovery from Leached Electronic Waste. Leached electronic waste samples were diluted with ultrapure water in a ratio of 1:1 before gold recovery was performed. The recovery process was conducted by adding $50 \mathrm{mg}$ of the $\mathrm{N} 12$ powder into the centrifuge tube containing $10 \mathrm{~mL}$ of the solution. The container was sealed, and the sample was agitated for $4 \mathrm{~h}$ using Stuart SF1 at 500 osc $\mathrm{min}^{-1}$ at room temperature. Samples were filtered (Whatman 41) to remove the nylon-12.

Capacity of the Material. Solution containing $200 \mathrm{ppm}$ of $\left[\mathrm{AuCl}_{4}\right]^{-}$in $5 \%$ hydrochloric acid was prepared by diluting the PerkinElmer spectroscopy standard of gold (1000 ppm). Ten milliliters of the solution was placed in a $15 \mathrm{~mL}$ centrifuge tube, and $25 \mathrm{mg}$ of nylon-12 was added. The tube was sealed and agitated using Stuart SF1 at $500 \mathrm{osc} \mathrm{min}^{-1}$ for $24 \mathrm{~h}$ at room temperature. Samples were filtered (Whatman 41) to remove the nylon-12. A similar test was performed for small, 3Dprinted, rectangle-shaped solid objects having average mass of 8 mg. Additionally, the capacity of slightly larger 3D-printed objects, with an average mass of $131 \mathrm{mg}$, was tested by reacting the objects with $50 \mathrm{~mL}$ of the synthetic solution used to test the capacity of the nylon-12 powder.

Recovery from Synthetic Solution Using 3D-Printed Mesh. The test conducted using 3D-printed mesh cube was done by contacting the synthetic solution containing $100 \mathrm{ppm}$ of $\mathrm{Ni}, \mathrm{Zn}, \mathrm{Fe}$, and $\mathrm{Cu}$ and $50 \mathrm{ppm}$ of $\mathrm{Al}, \mathrm{Cr}, \mathrm{Pb}$, and $\mathrm{Sn}$ along with $5 \mathrm{ppm}$ of $\mathrm{Pd}, \mathrm{Pt}$, and $\mathrm{Au}$ in $5 \%$ hydrochloric acid with the object. Twenty milliliters of the synthetic solution was placed in a $50 \mathrm{~mL}$ centrifuge tube containing the mesh. The tube was sealed and agitated using Stuart SF1 at 500 osc $\min ^{-1}$ for $4 \mathrm{~h}$ at room temperature. Recovery percentages of the metals that had significant decrease in concentration are presented in Table S2.

Recovery from Synthetic Solution Using 3D-Printed Column. The test conducted using 3D-printed columns was done by letting synthetic solution containing $100 \mathrm{ppm}$ of $\mathrm{Ni}$, $\mathrm{Zn}, \mathrm{Fe}$, and $\mathrm{Cu}$ and $50 \mathrm{ppm}$ of $\mathrm{Al}, \mathrm{Cr}, \mathrm{Pb}$, and $\mathrm{Sn}$ along with 5 ppm of $\mathrm{Pd}, \mathrm{Pt}$, and $\mathrm{Au}$ in 5\% hydrochloric acid flow through the column. A $10 \mathrm{~mL}$ sample size was used for the tests. The length of the column was varied. In initial tests, a column containing one functional middle piece with the length of $35 \mathrm{~mm}$ and the diameter of $27 \mathrm{~mm}$ was used. To further improve the recovery, following tests were done utilizing a column with three functional middle pieces attached and a total length of $105 \mathrm{~mm}$ (Figure S2a). Additionally, a column with 10 functional middle pieces and total length of $350 \mathrm{~mm}$ was tested (Figure S2b). For the $350 \mathrm{~mm}$ long column, a sample size of $20 \mathrm{~mL}$ was used. The size of the column can be easily scaled, as illustrated in Figure S2c. Recovery percentages of the metals that had significant decrease in concentration are presented in Table S2.

Removal of Gold from Scavenger Objects. Gold removal tests were conducted using a 3D-printed column consisting of three functional units (Figure S2a). First, the column was loaded by letting $10 \mathrm{~mL}$ of $5 \%$ hydrochloric acid solution, containing $10 \mathrm{ppm}$ of $\mathrm{Au}$, run through the column. This loading solution was prepared by diluting the $1000 \mathrm{ppm}$ PerkinElmer spectroscopy standard of gold. About 30\% nitric acid (prepared by diluting nitric acid so that the concentration of the removal eluent was $45 \%$ of the concentration of concentrated nitric acid) was then used as the gold removal eluent. The removal eluent was let to flow through the collector column in $15 \mathrm{~mL}$ pulses. The first $15 \mathrm{~mL}$ pulse recovered $47.9 \%$ of the adsorbed gold. By using four $15 \mathrm{~mL}$ pulses, $99 \%$ of the adsorbed gold was recovered. To confirm the ability to use organic eluents, a similar elution test was carried out by using diethylene glycol dibutyl ether as the removal eluent. With one $15 \mathrm{~mL}$ pulse, about $15 \%$ of gold was recovered. By repeating the pulses, the recovery percentage could be improved, as in the case of nitric acid eluent.

Three-dimensional Printing. Designing of the 3D-printed objects was done using FreeCAD v0.16. Designed objects were prepared for the printer using Slic3r v1.2.9. Images of the designed pieces are presented in Figures S3-S6. Threedimensional printing was done by using ShareBot SnowWhite SLS 3D printer. Nylon-12 was used as printing powder. A layer thickness of $0.1 \mathrm{~mm}$ was used for printing the objects. The laser power used was $50-55 \%$, with a rate of $64000\left(2560 \mathrm{~mm} \mathrm{~s}^{-1}\right)$. The build plate temperature was set to $173{ }^{\circ} \mathrm{C}$ and was changed to $161{ }^{\circ} \mathrm{C}$ at an object height of $0.5 \mathrm{~mm}$. The environment temperature was set to $144{ }^{\circ} \mathrm{C}$. Five warming layers were used for the print, and the wait time between each layer was set to 12 s.

Objects were cleared thoroughly of any unsintered powder remaining on the surfaces before being taken for adsorption tests. The column used for the tests consisted of several pieces that could be linked together to form the actual column.

ICP-OES Measurements. Measurements were done with PerkinElmer ICP-OES Optima 8300 DV using a GemCone low flow nebulizer and a cyclonic spray chamber. For all measurements, argon gas flow of $8 \mathrm{~L} \mathrm{~min}^{-1}$, nebulizer gas flow of $0.6 \mathrm{~L} \mathrm{~min}^{-1}$, auxiliary gas flow of $0.2 \mathrm{~L} \mathrm{~min}^{-1}$, and a sample flow rate of $1.5 \mathrm{~L} \mathrm{~min}^{-1}$ was used. The radio frequency power used for all of the measurements was $1500 \mathrm{~W}$. Method detection limits (MDLs) were established using the United 
States Environmental Protection Agency method 200.7. ${ }^{34}$ The metals analyzed, MDLs, chosen wavelengths, calibration ranges, and $R$ values for methods used for synthetic solution and for leached electronic waste are presented in Tables S3 and S4.

Concentrations of the metals present in the solutions were measured before and after the recovery process. Metal recovery rates were calculated from the change in concentration

$$
\text { recovery }(\%)=\frac{c_{\mathrm{i}}-c}{c_{\mathrm{i}}} \times 100
$$

where $c_{\mathrm{i}}$ is the concentration of the analyte before the gold recovery process and $c$ is the concentration of the analyte after the recovery.

SEM. Scanning electron microscope measurements were done using Zeiss EVO-50XVP. Three-dimensionally printed objects were cleared thoroughly of any unsintered powder before the SEM measurements. Figure S1 shows the surface of a 3D-printed object that was manufactured using high laser power.

Statistical Analysis. Results were statistically analyzed on a confidence interval of $95 \%$. The analysis was done as follows

$$
t=\frac{|\bar{x}-\mu|}{s / \sqrt{n}}
$$

where $\bar{x}$ is the average of the analyte concentration after the recovery process. $\mu$ is the average of the analyte concentration before the recovery process. $s$ is the standard deviation of the analyte concentration after the recovery process and $n$ is the number of replicates. The number of replicates used for all experiments was three. The hypothesis of the $t$-tests was that whether the concentrations after the recovery process resulted in significantly lower concentrations than those of the original sample; thus, the one tailed $t$-test was performed.

\section{ASSOCIATED CONTENT}

\section{S Supporting Information}

The Supporting Information is available free of charge on the ACS Publications website at DOI: 10.1021/acsomega.7b01215.

Metal recovery percentages; ICP-OES measurement parameters; SEM figure; figures of the scavenger columns; figures of the designed columns (PDF)

\section{AUTHOR INFORMATION}

\section{Corresponding Authors}

*E-mail: ari.o.vaisanen@jyu.fi (A.V.).

*E-mail: kari.t.rissanen@jyu.fi (K.R.).

*E-mail: matti.o.haukka@jyu.fi (M.H.).

\section{ORCID}

Rajendhraprasad Tatikonda: 0000-0003-1277-3492

Kari Rissanen: 0000-0002-7282-8419

Matti Haukka: 0000-0002-6744-7208

\section{Author Contributions}

M.H., A.V., and K.R. conceptualized the use of scavenging materials, the design, and $3 \mathrm{D}$ printing of the reported scavengers, and supervised the characterization work. E.L. carried out the adsorption tests and metal-ion analytics; E.L. and L.K. prepared the 3D models of the scavenger and performed the $3 \mathrm{D}$ printing. R.T. did the SEM measurements. E.L, L.K, A.V., K.R., and M.H. wrote the manuscript.

\section{Notes}

The authors declare no competing financial interest.

\section{ACKNOWLEDGMENTS}

The research reported in this publication was solely performed at the Department of Chemistry, University of Jyväskylä, Finland, and was supported by the funding from Centennial Foundation of Technology Industries of Finland and Jane and Aatos Erkko foundation as a part of The Future Makers program. The research was also supported by the Academy of Finland (grant nos: 295581 (M.H.), 263256, 265328, and 292746 (K.R.)) and the University of Jyväskylä.

\section{REFERENCES}

(1) Gross, B. C.; Erkal, J. L.; Lockwood, S. Y.; Chen, C.; Spence, D. M. Evaluation of $3 \mathrm{D}$ Printing and Its Potential Impact on Biotechnology and the Chemical Sciences. Anal. Chem. 2014, 86, 3240-3253.

(2) Yue, J.; Zhao, P.; Gerasimov, J. Y.; van de Lagemaat, M.; Grotenhuis, A.; Rustema-Abbing, M.; van der Mei, H. C.; Busscher, H. J.; Herrmann, A.; Ren, Y. 3D-Printable Antimicrobial Composite Resins. Adv. Funct. Mater. 2015, 25, 6756-6767.

(3) Seo, J.; Kushner, D. I.; Hickner, M. A. 3D Printing of Micropatterned Anion Exchange Membranes. ACS Appl. Mater. Interfaces 2016, 8, 16656-16663.

(4) Philamore, H.; Rossiter, J.; Walters, P.; Winfield, J.; Ieropoulos, I. Cast and 3D Printed Ion Exchange Membranes for Monolithic Microbial Fuel Cell Fabrication. J. Power Sources 2015, 289, 91-99.

(5) Nadgorny, M.; Xiao, Z.; Chen, C.; Connal, L. A. ThreeDimensional Printing of $\mathrm{pH}$-Responsive and Functional Polymers on an Affordable Desktop Printer. ACS Appl. Mater. Interfaces 2016, 8, 28946-28954.

(6) Coupland, D. R. Catalyst Manufacturing Method. U.S. Patent 9,278,338 B2, March 8, 2016.

(7) Tubío, C. R.; Azuaje, J.; Escalante, L.; Coelho, A.; Guitián, F.; Sotelo, E.; Gil, A. 3D Printing of a Heterogeneous Copper-Based Catalyst. J. Catal. 2016, 334, 110-115.

(8) Huber, C.; Abert, C.; Bruckner, F.; Groenefeld, M.; Muthsam, O.; Schuschnigg, S.; Sirak, K.; Thanhoffer, R.; Teliban, I.; Vogler, C.; Windl, R.; Suess, D. 3D Print of Polymer Bonded Rare-Earth Magnets, and 3D Magnetic Field Scanning with an End-User 3D Printer. Appl. Phys. Lett. 2016, 109, No. 162401.

(9) Kong, Y. L.; Tamargo, I. A.; Kim, H.; Johnson, B. N.; Gupta, M. K.; Koh, T.-W.; Chin, H.-A.; Steingart, D. A.; Rand, B. P.; McAlpine, M. C. 3D Printed Quantum Dot Light-Emitting Diodes. Nano Lett. 2014, 14, 7017-7023.

(10) Waste Electrical \& Electronic Equipment (WEEE), 2017. http://ec.europa.eu/environment/waste/weee/index_en.htm (accessed July 18, 2017).

(11) Kaya, M. Recovery of Metals and Nonmetals from Electronic Waste by Physical and Chemical Recycling Processes. Waste Manage. 2016, 57, 64-90.

(12) Akcil, A.; Erust, C.; Gahan, C. S.; Ozgun, M.; Sahin, M.; Tuncuk, A. Precious Metal Recovery from Waste Printed Circuit Boards Using Cyanide and Non-Cyanide Lixiviants-A Review. Waste Manage. 2015, 45, 258-271.

(13) Jadhav, U.; Hocheng, H. Hydrometallurgical Recovery of Metals from Large Printed Circuit Board Pieces. Sci. Rep. 2015, 5, No. 14574.

(14) Cayumil, R; Khanna, R.; Rajarao, R.; Mukherjee, P. S.; Sahajwalla, V. Concentration of Precious Metals during Their Recovery from Electronic Waste. Waste Manage. 2016, 57, 121-130.

(15) Donato, D. B.; Nichols, O.; Possingham, H.; Moore, M.; Ricci, P. F.; Noller, B. N. A Critical Review of the Effects of Gold CyanideBearing Tailings Solutions on Wildlife. Environ. Int. 2007, 33, 974984.

(16) Hilson, G.; Monhemius, A. J. Alternatives to Cyanide in the Gold Mining Industry: What Prospects for the Future? J. Cleaner Prod. 2006, 14, 1158-1167.

(17) Alguacil, F. J.; Adeva, P.; Alonso, M. Processing of Residual Gold(III) Solutions via Ion Exchange. Gold Bull. 2005, 38, 9-13. 
(18) Kim, E.-y.; Kim, M.-s.; Lee, J.-c.; Pandey, B. D. Selective Recovery of Gold from Waste Mobile Phone PCBs by Hydrometallurgical Process. J. Hazard. Mater. 2011, 198, 206-215.

(19) Yue, C.; Sun, H.; Liu, W.-J.; Guan, B.; Deng, X.; Zhang, X.; Yang, P. Environmentally Benign, Rapid, and Selective Extraction of Gold from Ores and Waste Electronic Materials. Angew. Chem., Int. Ed. 2017, 56, 9331-9335.

(20) Cui, J.; Zhang, L. Metallurgical Recovery of Metals from Electronic Waste: A Review. J. Hazard. Mater. 2008, 158, 228-256.

(21) Tuncuk, A.; Stazi, V.; Akcil, A.; Yazici, E. Y.; Deveci, H. Aqueous Metal Recovery Techniques from E-Scrap: Hydrometallurgy in Recycling. Miner. Eng. 2012, 25, 28-37.

(22) Trochimczuk, A. W. Uptake of Gold from Hydrochloric Acid Solutions by Polymeric Resins Bearing Various Phosphorus Containing Ligands. Sep. Sci. Technol. 2002, 37, 3201-3210.

(23) Fujiwara, K.; Ramesh, A.; Maki, T.; Hasegawa, H.; Ueda, K. Adsorption of Platinum(IV), Palladium(II) and Gold(III) from Aqueous Solutions onto L-Lysine Modified Crosslinked Chitosan Resin. J. Hazard. Mater. 2007, 146, 39-50.

(24) Ramesh, A.; Hasegawa, H.; Sugimoto, W.; Maki, T.; Ueda, K. Adsorption of gold(III), platinum(IV) and palladium(II) onto Glycine Modified Crosslinked Chitosan Resin. Bioresour. Technol. 2008, 99, 3801-3809.

(25) Parajuli, D.; Khunathai, K.; Adhikari, C. R.; Inoue, K.; Ohto, K.; Kawakita, H.; Funaoka, M.; Hirota, K. Total Recovery of Gold, Palladium, and Platinum Using Lignophenol Derivative. Miner. Eng. 2009, 22, 1173-1178.

(26) Pilśniak, M.; Trochimczuk, A. W.; Apostoluk, W. The Uptake of Gold(I) from Ammonia Leaching Solution by Imidazole Containing Polymeric Resins. Sep. Sci. Technol. 2009, 44, 1099-1119.

(27) Kang, E. T.; Ting, Y. P.; Neoh, K. G.; Tan, K. L. Electroless Recovery of Precious Metals from Acid Solutions by N-Containing Electroactive Polymers. Synth. Met. 1995, 69, 477-478.

(28) Neoh, K. G.; Tan, K. K.; Goh, P. L.; Huang, S. W.; Kang, E. T.; Tan, K. L. Electroactive polymer- $\mathrm{SiO}_{2}$ Nanocomposites for Metal Uptake. Polymer 1999, 40, 887-893.

(29) Neoh, K. G.; Young, T. T.; Looi, N. T.; Kang, E. T.; Tan, K. L. Oxidation-Reduction Interactions between Electroactive Polymer Thin Films and $\mathrm{Au}(\mathrm{III})$ Ions in Acid Solutions. Chem. Mater. 1997, 9, 2906-2912.

(30) Reck, B. K.; Graedel, T. E. Challenges in Metal Recycling. Science 2012, 337, 690-695.

(31) Haukka, M.; Väisänen, A.; Rissanen, K.; Lahtinen, E.; Kivijärvi, L. A Porous Body, Method for Manufacturing It and Its Use for Collecting Substance from Source Material. Finland Patent Application no. 20175652, 2017.

(32) Cheval, N.; Gindy, N.; Flowkes, C.; Fahmi, A. Polyamide 66 Microspheres Metallised with in Situ Synthesised Gold Nanoparticles for a Catalytic Application. Nanoscale Res. Lett. 2012, 7, 182.

(33) Doidge, E. D.; Carson, I.; Tasker, P. A.; Ellis, R. J.; Morrison, C. A.; Love, J. B. A Simple Primary Amide for the Selective Recovery of Gold from Secondary Resources. Angew. Chem., Int. Ed. 2016, 55, 12436-12439.

(34) Martin, T. D.; Brockhoff, C. A.; Creed, J. T.; Long, S. E. Determination of Metals and Trace Elements in Water and Wastes by Inductively Coupled Plasma-Atomic Emission Spectrometry. Methods for the Determination of Metals in Environmental Samples; CRC Press Inc.: Boca Raton, 1992; pp 33-91. 